\title{
Software for generating auto-random-dot stereograms
}

\author{
DAVID G. STORK \\ Stanford University, Stanford, California \\ and \\ CHRIS ROCCA \\ Clark University, Worcester, Massachusetts
}

\begin{abstract}
We present a brief description of the theory behind Tyler's (1983) auto-random-dot stereogram (ARDS) technique, and compare some of its strengths and drawbacks with those of standard random-dot stereograms (Julesz, 1964). We give in algorithmic form a computer program for generating ARDSs, as well as some extensions of the basic technique. A program for generating ARDSs from black and white user-drawn graphic images is available for the Macintosh personal computer.
\end{abstract}

Random-dot stereograms, introduced by Julesz (1964), consist of two images (one for each eye) of dots placed in what appears to be a random arrangement. When either of the component images is viewed individually, no depth percept results. If, however, the two images are stereoscopically fused, a three-dimensional texture pattern can be seen. Whereas in classical stereograms, many depth cues are present simultaneously-size, perspective, shading, occlusion, and so forth-the sole depth cue in random-dot stereograms is binocular disparity. As such, these random-dot stimuli (and more generally, cyclopean stimuli; see Julesz, 1971) have been extremely useful in elucidating the nature and sequence of early visual functions.

The three primary components in a cyclopean stimulus are the inducing elements, the three-dimensional depth pattern desired, and the technical method of presentation. Inducing elements have most commonly included black and white dots, including dynamically flickered dots (e.g., Julesz \& Chang, 1976; Neff, Schwartz, \& Stork, 1985), as well as vernier acuity stimuli (Julesz \& Spivack, 1967), line segments, typed letters, and so forth. A wide variety of depth patterns have been utilized in perceptual research, including simple geometric figures and traditional illusions, sinusoidal disparity gratings (Tyler, 1974; Tyler \& Raibert, 1975), and many others.

There is a long history of technical methods of presentation of stereoimages-from Wheatstone's stereoscope to modern stereoholographic techniques-that is too extensive to recount here. Okoshi (1976) presents a history and description of the principles underlying a wide range of these stereoimaging methods, including many that have

This research was supported in part by NIH-NEI Grant EY-05342 to David G. Stork. We wish to extend our many thanks to Jim Crowell and Ian Aberbach for computer work. Reprint requests should be addressed to David G. Stork, Departments of Psychology and Electrical Engineering, Jordan Hall, Stanford Universisty, Stanford, CA 94305. not been used extensively by perceptual investigators. The most common technical methods for separating the two component images of a stereogram (standard or randomdot) in perceptual and behavioral research are:

1. Red-green anaglyph systems, in which the two component stereogram images are presented in red and in green, superimposed (either on a color TV monitor or on the printed page). The subject views the pattern through a red filter over one eye, and a green one over the other, thereby ensuring optical separation of the images.

2. Standard stereo viewers, in which prism lenses permit each eye to see its appropriate image, optically superimposed.

3. Wheatstone stereoscopes, in which a mirror system permits the optical superposition of the two component images.

4. Polaroid systems, in which the two component images are projected through linear polarizing filters (oriented orthogonally to each other) onto a polarizationpreserving screen. The subject places a polaroid filter of the appropriate orientation over each eye, thereby to see only one component image in each eye.

5. Free viewing, in which the component images are printed side by side, and the subject converges sufficiently so that each image falls on the corresponding positions on the two retinas.

The drawback of the presentation techniques listed above, except for the last, is the need for special equipment such as lenses, projectors, or polarization-preserving screens. The drawback of free viewing, on the other hand, is that it can cause subject discomfort because of unnatural convergence requirements. A closely related drawback is that the solid visual angle subtended by the free-viewed stereogram must be small, again because of convergence and accommodation limitations (see below).

In 1983, Tyler presented an auto-random-dot stereogram (ARDS) that surmounted some of the problems of free viewing; Figure 1 shows such an ARDS, from Falk, 


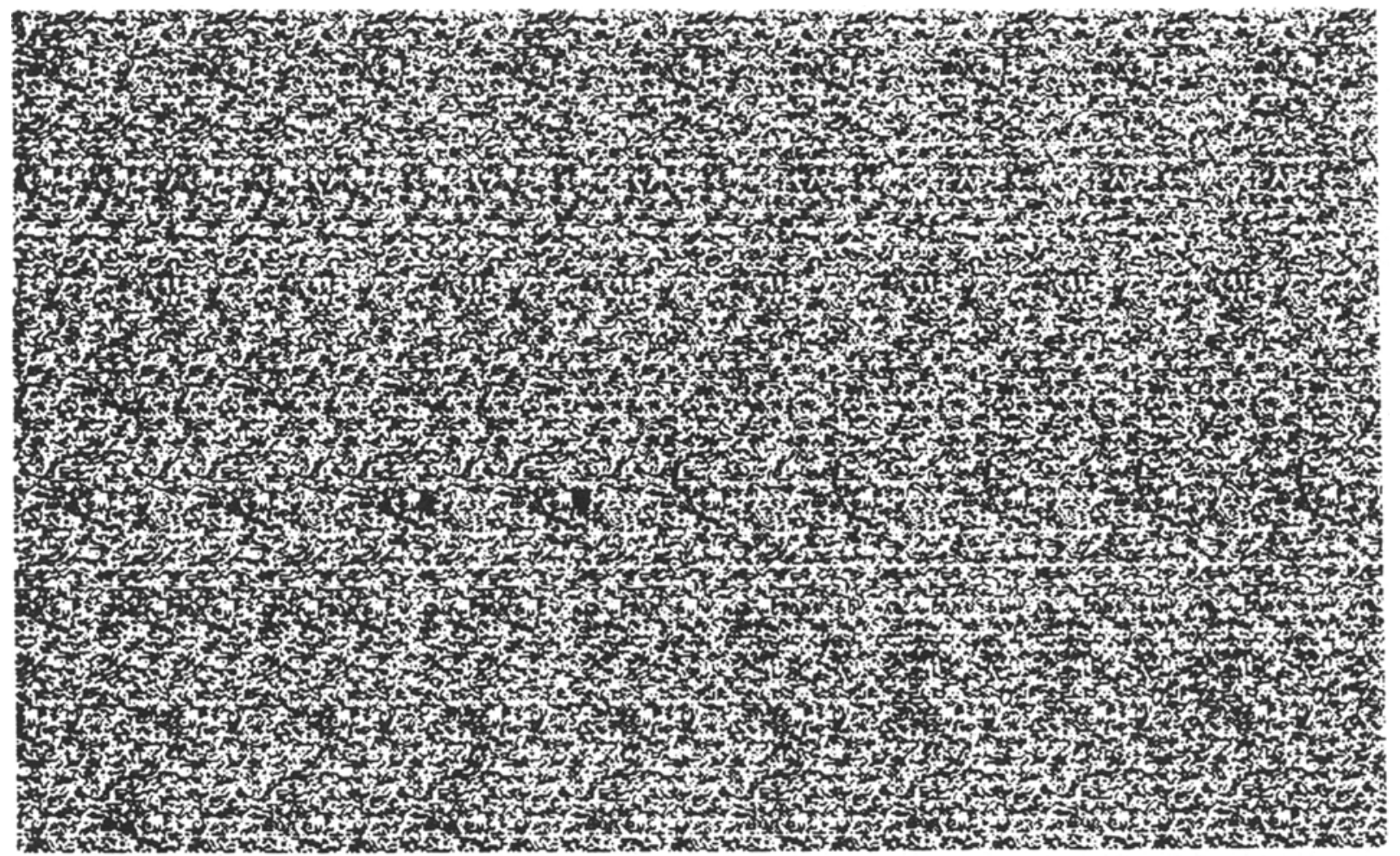

Figure 1. Auto-random-dot stereogram. If one free-fuses the two large dark dots under crossed or hyperconvergent conditions (i.e., the right eye foveates the left dot, and vice versa), a raised message will emerge; under uncrossed or hypoconvergent conditions (i.e., the right eye foveates the right dot, and the left eye the left dot), a raised sheet will appear, with the letters cut through. ${ }^{1}$

Brill, and Stork (1988). The technique is called auto-RDS because the single image serves as its own right and left component images-each printed dot appears in both the right and the left eyes' views.

\section{Benefits of ARDSs as Opposed to Standard RDSs}

ARDSs have some advantages over standard RDSs, but also have some drawbacks. Neither of these techniques requires any equipment for viewing, but only in autoRDSs can images of arbitrarily large visual angle be portrayed (as long as the image is not a rare pathological case; see below). Standard RDSs (when viewed according to the traditional crossed convergence method) are limited roughly to the subject's maximum convergence angleperhaps $15^{\circ}$ left to right in the frontal plane (Figure 2). Note that this result is nearly independent of the viewing distance; for a large viewing distance, the images in a standard RDS can be made larger, but they nevertheless cannot subtend a left-to-right angle greater than roughly the maximum convergence angle.

In an informal poll of 5 experienced free-fusing subjects, all could properly fuse and perceive depth in the original for Figure 1 (19.2 cm left to right) from the standard near-point distance of $25 \mathrm{~cm}$, thereby giving a leftto-right visual angle of roughly $44^{\circ}$. From the same viewing distance, none of these subjects could converge to fuse standard side-by-side stereograms subtending this same $44^{\circ}$. (These subjects could only fuse the side-by-side stereogram if the viewing distance was increased greatly, but then, of course, the visual angle subtended was correspondingly reduced. It seems unlikely that any but the most highly trained observer could hyperconverge sufficiently to be able to experience depth in a standard RDS subtending a left-to-right visual angle of as large as $44^{\circ}$.)

In normal vision, convergence and accommodation are typically yoked; that is, when a subject looks at an object at a given distance, both convergence and accommodation are appropriate for that distance. This is not the case for a standard RDS, as is shown in Figure 2 (top): subjects are forced to converge to one distance, but accommodate to another. Subjects often complain that whereas they have converged properly to fixate the fixation dots, the image appears blurry because of improper accommodation. Although this problem remains in an auto-RDS, it can be reduced, because the fixation dots can be placed closer together than in a standard RDS subtending the same visual angle. Figure 2 (bottom) illustrates how the convergence and accommodation can be made more nearly equal in an ARDS than for a corresponding RDS.

Whereas an arbitrary depth contour can be portrayed in a standard RDS, a drawback of the ARDS technique is that there are some patterns that cannot effectively be portrayed (see below). 

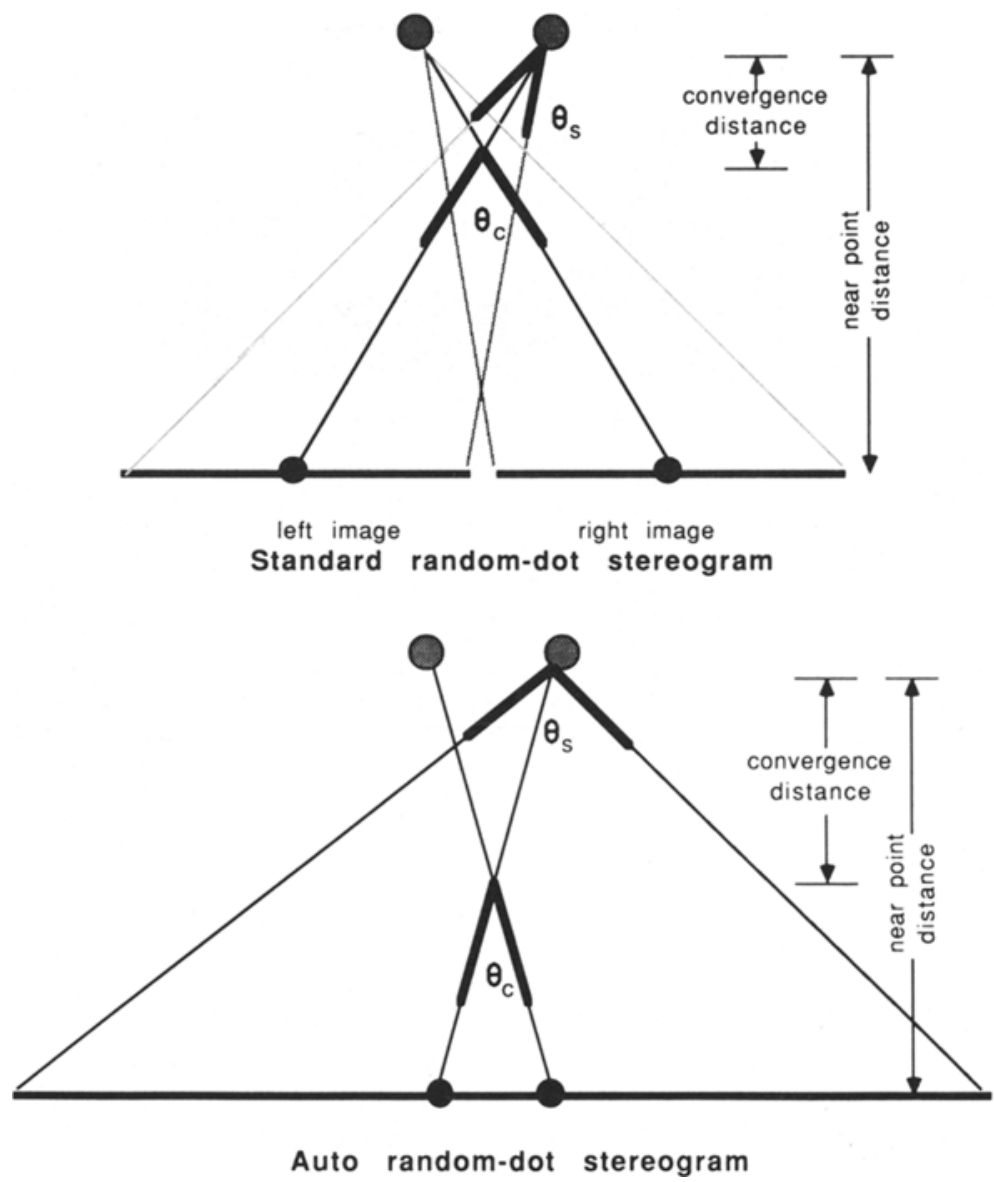

Figure 2. The visual angle $\theta_{\mathrm{s}}$ that can be subtended by a standard free-fused RDS is limited by the convergence angle, $\theta_{c}$, whereas the visual angle for an auto-RDS is not. Top: Schematic diagram of two eyes viewing standard random-dot stereogram. Bottom: Auto-random-dot stereogram. The large black dots represent fixation markers. Note that the distances of convergence and of accommodation are more nearly equal in the ARDS than in the standard side-by-side RDS. This effect is even more pronounced when the standard RDS subtends the same visual angle as the ARDS does.

\section{Theory of the ARDS}

We present here the theory underlying Tyler's ARDSs, so that readers can understand the strengths and limitations of the technique, and so that those who cannot take advantage of the Macintosh program offered below can perhaps write their own programs for generating ARDSs. Figure 3 shows a schematic of an ARDS. It consists of a single random-dot image, with two fixation markers separated by the convergence distance (measured in multiples of the unit cell). In order to understand the operation of an ARDS, consider first a single horizontal row of dots. During free fusing of the fixation markers, each eye sees this row of dots, but they are in effect shifted horizontally relative to each other, due to the convergence (Figure 3, bottom). That is, as far as the cortical stage computing depth from binocular disparity is concerned, there are in effect two rows of dots. The two rows could be considered equivalent to those representing each eye's respective view in a standard RDS, but there is one cru- cial difference. Because each image is physically separate in a standard RDS, the value (white or black) of each cell in the right eye's image can be assigned independently of that in the left eye's view. This is not so in an autoRDS; whatever cell value is assigned to a cell for one eye must appear in the other eye's view, but it will be displaced by the convergence distance.

Figure 4 shows, in greater detail, a split representation for a single line of an auto-RDS, for a convergence distance arbitrarily chosen to be four cells. (In a typical ARDS, the convergence distance will be much greater, perhaps 40 or 50 of the smaller cells.) The individual cells are marked $1,2,3,4,5,6, \ldots$ Values are assigned to the cells, starting at the left and progressing to the right. Suppose the desired depth pattern requires that this entire row appear at the same intermediate depth, as is shown schematically in Figure 4a. The first four cells, as considered by the left eye's view, do not have companion cells in the right eye's view. These four cells will then be rival- 


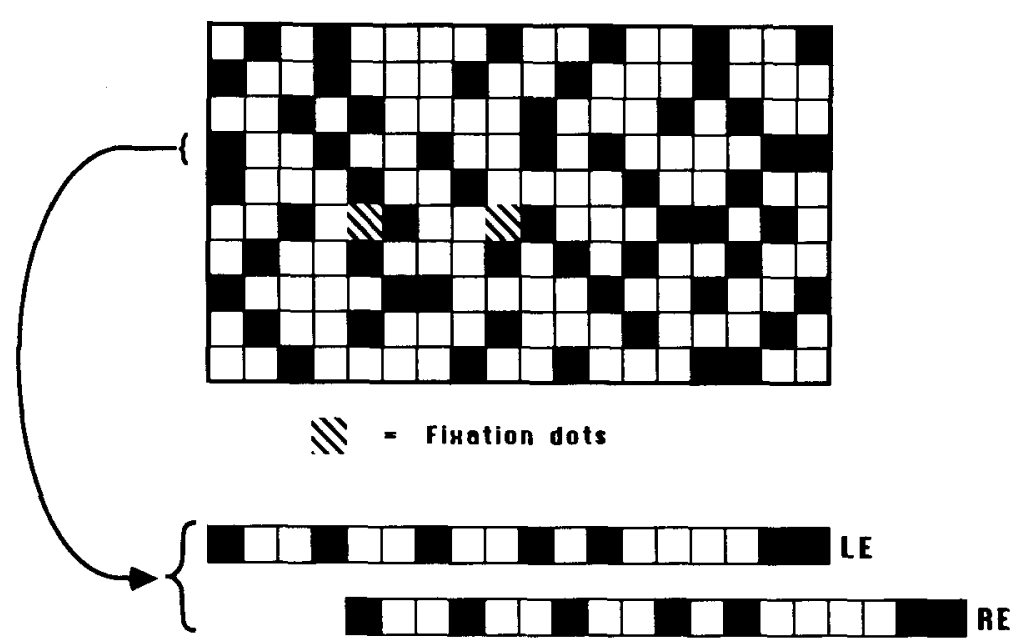

Figure 3. Schematic of an ARDS. The cells are drawn quite large, solely for illustration purposes. A single horizontal row of dots in the ARDS is shown split annd displaced horizontally by the convergence distance (here, four cells), to represent the two effective rows, as presented to each eye.

rous with the area of the page outside the ARDS proper. This rivalry is irrelevant for the portrayal of our depth pattern. The first four cells can each be assigned any value; they are thus labeled $1,2,3,4$, as shown. Later they shall be "painted" black or white, depending on the assigned values. In an ARDS, this assignment demands that the first four cells in the right eye's view also be 1 , $2,3,4$, since they are, after all, the same cells on the printed page, merely displaced by the convergence distance (Figure 4b).

Consider now Cell 5 , as viewed by the left eye. For the desired depth pattern to be displayed, this cell must have a companion at the same effective position (zero relative disparity in this representation), since our goal is to portray a line at a given depth. Hence the value 1 is assigned to Cell 5, as shown in Figure 4c. (Consequently,

a)
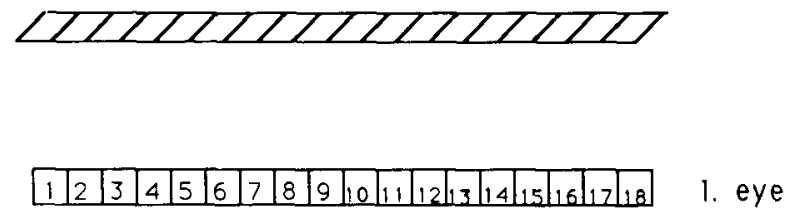

b)

\begin{tabular}{|l|l|l|l|l|l|l|l|l|l|l|l|l|l|l|l|l|l|}
\hline 1 & 2 & 3 & 4 & 5 & 6 & 7 & 8 & 9 & 10 & 11 & 12 & 13 & 14 & 15 & 16 & 17 & 18 \\
\hline
\end{tabular}

c)

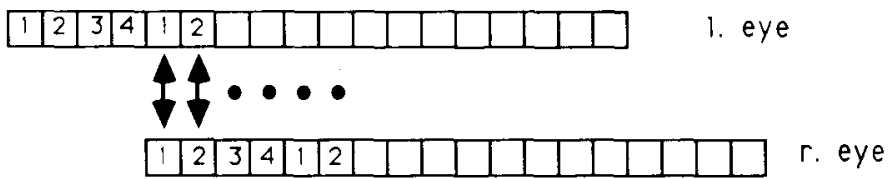

d)

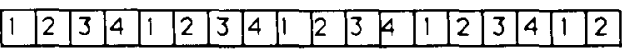

Figure 4. (a) Perspective view of the desired depth profile to be portrayed in the row: a single horizontal line of uniform depth. (b) The single line is presented split and arbitrarily displaced vertically to show the right and left eyes' images as at the bottom of Figure 3. (c) The fifth cell, as considered in the left eye's view, must be assigned the same value as the one "beneath" it in the right eye's view (i.e., a 1). (d) The final assignment of the cells in the row. 
a)

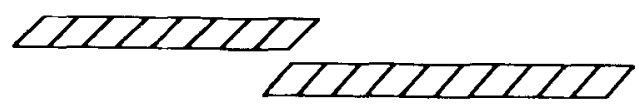

b)

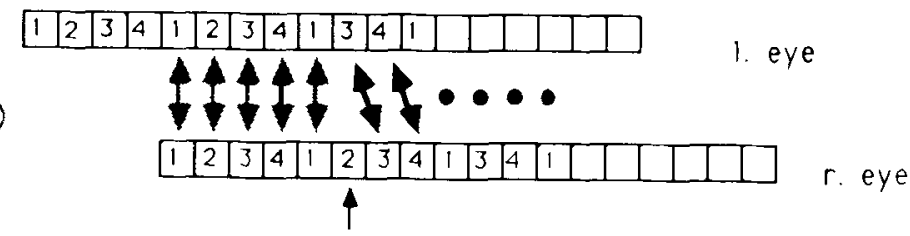

c)

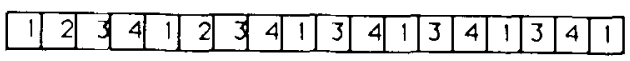

Figure 5. (a) Perspective view of the desired depth profile to be portrayed in the line-a depth discontinuity, with greater depth on the right side. (b) The first nine cells are filled as in Figure 4. Cell 10, however, must correspond to the cell displaced one step to the right in the right eye's view. This cell (Cell 7 ) has already been assigned the value 3 . Hence, Cell 10 must be assigned the value 3 as well. Likewise, Cell 11 must be assigned a 4 , and so on. (c) The finsl assignment of the cells in the row.

this value then appears in the same cell in the right eye's view.) Progressing to the right, each cell considered in the left eye's view is set to be the same as that represented directly "beneath" it in the right eye's, thereby yielding the repeating pattern $1,2,3,4,1,2,3,4, \ldots$.

All cells labeled " 1 " are then given the same color (black or white); all cells labeled " 2 "' are given the same color but one chosen independently from that for cells labeled " 1 ," and so forth (but see below). The percentage of white cells can be set by the experimenter, but it should not be too high or too low; if all cells are assigned white or all black, for instance, the "stereogram" becomes useless.

When the ARDS line of Figure $4 \mathrm{~d}$ is viewed with a convergence distance of four cells, each eye receives the same pattern; there is, effectively, no change in disparity, and hence the stereoimage appears flat. This is a more complicated version of the familiar wallpaper illusion, which can also be illustrated by misfusing vertical bars, say of a cage or of a repetitive tile pattern on a bathroom floor. Each bar of the cage then appears to be equally distant from the viewer.

a)

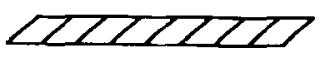

a)
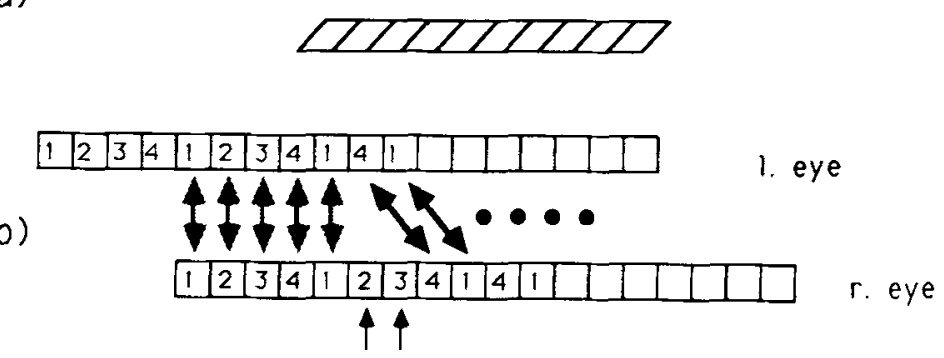

c)

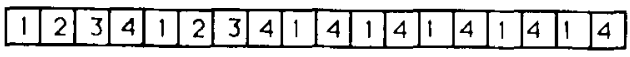

Figure 6. (a) As in Figure 5, but with a disparity change twice as great as that in the step of that figure. (b) Cells 1-9 are assigned as in Figures 4 and 5. Cells to the right of Cell 9 are assigned according to a displacement of two steps to the right. (c) The final assignment of the cells in the row. 
a)
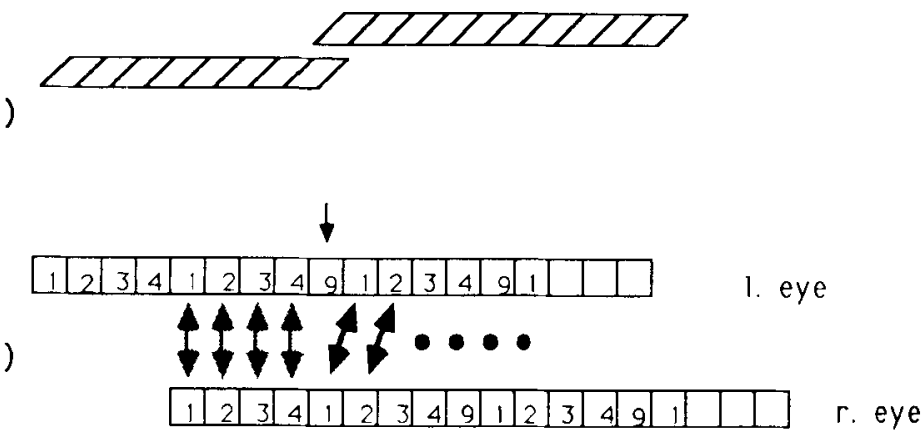

c)

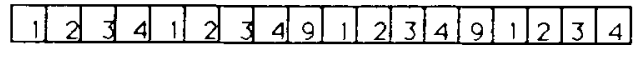

Figure 7. (a) As in Figure 6, but a step up by a disparity change corresponding to one cell. (b) Cells 1-8 are assigned as above. Cell 9 has no companion and is hence "free" (i.e, set to a new, unused value, here 9). (c) The final assignment of the cells in the row.

Now suppose that on a different line of the ARDS the desired depth pattern contains a disparity change across the line, here such that the left side will appear closer than the right (Figure 5a). The leftmost cells should be assigned values in the same manner as above (Figure $4 a-c$ ) to yield one segment of depth at the left. At the position of the disparity step (here Cell 10), that cell does not correspond to the one "beneath" it in the right eye's view, but instead to the cell displaced one cell to the right in the right eye's view (Figure 5b). Hence, Cell 10 is assigned the value 3 (not 2). Likewise, Cell 11 should correspond to the cell one step to its right in the right eye's view (the value 4), and so on (Figure 5c). Each subsequent cell is paired with the one displaced by one cell. The resulting sequence, $1,2,3,4,1,2,3,4,1,3,4,1,3,4, \ldots$, when free-fused with a convergence distance of 4 cells, will portray the step as in Figure 5a. Note that Cell 6 (assigned a 2) in the right eye's view has no companion, as we expect from Figure 5a: there are parts of the perceptual surface apparent to only one (here the right) eye.

If the desired step in depth is twice as large, then the correlation will be displaced by two cells (Figure 6a) and there will be two unpaired cells in the right eye's view, as if the subject is seeing behind a surface even higher than in Figure 5.

Suppose that on a different row of the ARDS the desired depth pattern requires a step in the opposite direction; the right side of the row must appear closer than the left (Figure 7a). In this case, the first eight cells are assigned as described above. At Cell 9, however, the unpaired cell must be in the left eye. Thus Cell 9 is effectively skipped, and the correlations are made in the direction opposite those in Figure 5. Cell 9 can be assigned a value unused before, which is shown here as 9 .
A typical row in a stereoimage will consist of several jumps in disparity; the technique described above is applied throughout. To understand the method in greater detail, imagine a computational device for assigning the values in any row as follows (Figure 8): The device starts at the left; its arrowhead is first set at Cell 1. Then the device reads the desired disparity from some data file containing the desired stereoimage. The device sets the pointer on its "disparity scale" accordingly; then it reads the value of the cell at the tail of the arrow, and assigns it to the cell at the arrow's head. If the tail of the arrow is to the left of the leftmost cell (Cell 1), it assigns a new value, different from any used previously.

\section{Some Limitations of the ARDS Technique}

There are some limitations in this method, due to mathematical constraints on the assignment of cell values. In

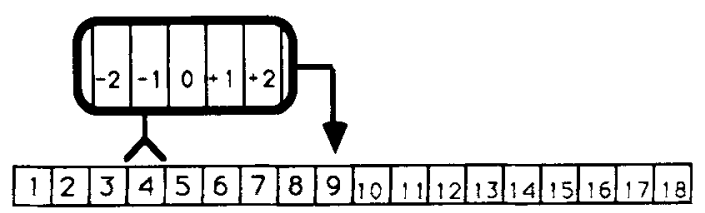

Figure 8. The algorithm can be understood by considering a conceptual "cell-assigning device" that has a pointer. The device starts from the left and moves to the right across a single row of the image. The device's arrowhead is placed at a given cell in the line, and the device reads the depth (disparity) from the experimenter's image or database (suitably coded). This disparity controls the position of the "tail" of the device, as marked. The device reads the cell value at the tail and fils it in at the cell at the head of the arrow. The device then moves one cell to the right and repeats the process. At the beginning, when the tail is off the image, the device fills each "free" cell with a new (i.e., previously unassigned) value. 
particular, not every three-dimensional image can be unambiguously portrayed in an ARDS. For example, suppose the crossing distance is 4 cells, and the desired depth pattern requires a step corresponding to a disparity change of four cells. Then the pattern along the row will be 1 , $2,3,4,1,2,3,4, \ldots$, just as in the case of a flat image; the image will be ambiguous. Such a depth pattern would not be ambiguous in a standard RDS. Consider Figure 5 too; the repeating pattern $1,4,1,4, \ldots$ at the right could be ambiguous, portraying a contour of relative disparity -2 , or 0 , or +2 , or ... cells.

A more serious drawback is that for any specified crossing distance, there exist desired depth contours that cannot be portrayed, because the constraints on the cell assignments cannot all be satisfied. Figure 9 illustrates a source of such limitations.

Suppose Cell 3 has been assigned a value, shown in Figure 9 as black. Suppose the desired depth pattern requires that for assigning Cell 5 , the disparity value on the
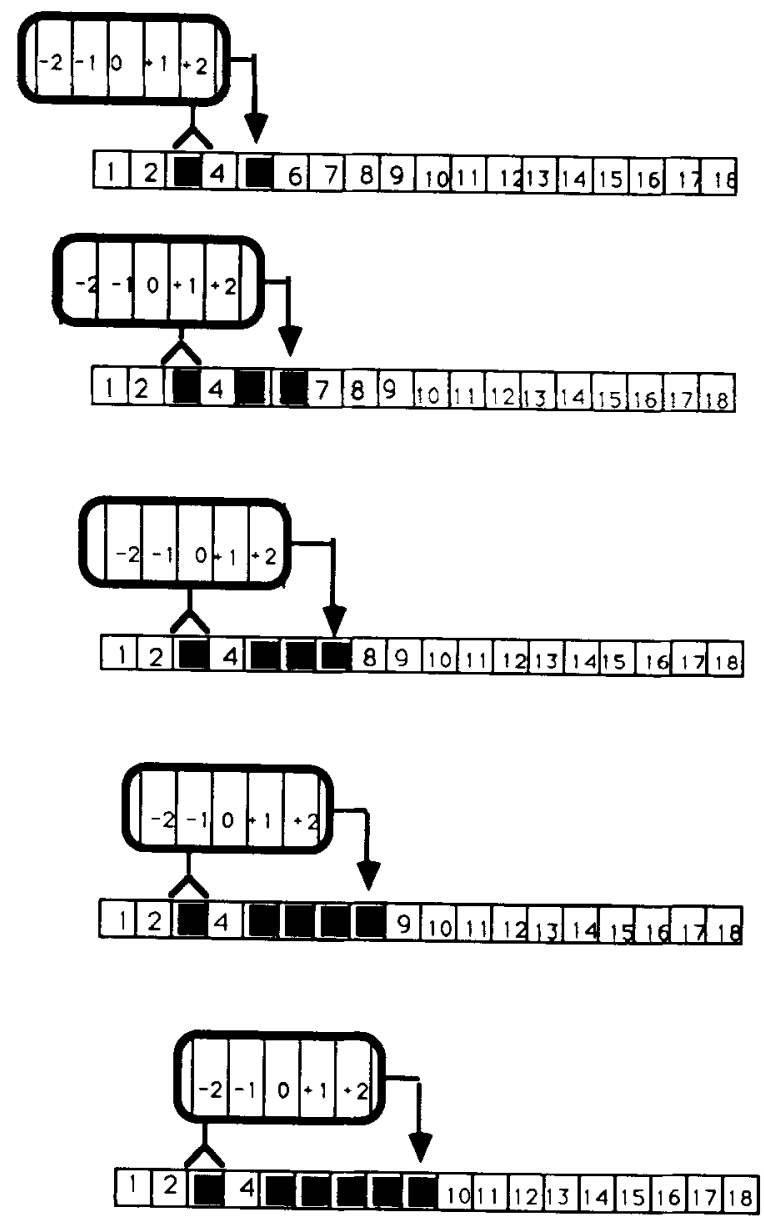

Figure 9. Certain pathological desired depth patterns cannot be portrayed in an ARDS. Here, Cells 5-9 have depths corresponding, respectively, to $+2,+1,0,-1$, and -2 on the cell assigning device, and thus they are all set equal to the value for Cell 3. If Cell 4 is never read, then the entire row to the right appears black, and hence portrays no depth. device is +2 ; for assigning Cell 6 , it is +1 ; for Cell 7 , it is 0 ; and so forth. In that case, all Cells 5-9 will be assigned black. If we continue across the row (and if Cell 4 is not required to be read), then the entire row will be black, and clearly our depth pattern will not be portrayed.

Although desired depth contours that cannot be portrayed in an ARDS are not plentiful, the mathematical construction necessary to determine whether a contour can or cannot be portrayed is arduous (see below). It is simplest just to try to make the ARDS. If necessary, the crossing distance may be increased, since the smaller the crossing distance, the more tightly constrained the assignments of the cells must be. There is no general guarantee that an increased crossing distance will permit the pathological image to be represented, however. Furthermore, if the crossing distance is increased substantially, the subject can no longer converge sufficiently and preserve the visual angle of the image.

\section{Variations}

There are several variations on the ARDS technique. Cells can be assigned letters or other symbols instead of just white and black dots, though with a decrease in resolution and contour complexity. Certain depth contours permit the same sequence of cell values at both the right of each row and at the left. In such cases, multiple copies of the ARDS can be placed contiguously, side by side, to make ARDS "wallpaper." Finally, Stork and Aberbach (1983) noted that under certain circumstances, a second image could be encoded in the orthogonal direction. A simple such ortho-ARDS is shown in Figure 10.

\section{Unsolved Problems}

There are two general unsolved problems in the theory of ARDSs.

First, as has been shown above, some desired depth contours can be portrayed with the ARDS technique, whereas others give ambiguous or unacceptable depth images. Is there an efficient means for mathematically determining whether a particular desired depth contour can or cannot be portrayed (for a given crossing distance), other than explicitly calculating the cell assignments? Is there a method (other than exhaustive search) for determining the minimum crossing distance for which a desired depth contour can be portrayed without ambiguities?

Second, whereas the cell-assignment constraints for an ARDS cannot always be satisfied for an arbitrary desired contour, the constraints in an ortho-ARDS are even more restrictive. Thus, it is rare that a pair of images can be represented in the orthogonal views of an ortho-ARDS. One class of images that can always be portrayed in an ortho-ARDS includes the following: each of the desired depth contours is solely a function of the vertical position (and is independent of horizontal position). Thus, for instance, a horizontal sine-wave grating and a horizontal square-wave grating of different disparity ranges and spatial frequencies can be portrayed in the orthogonal views. 


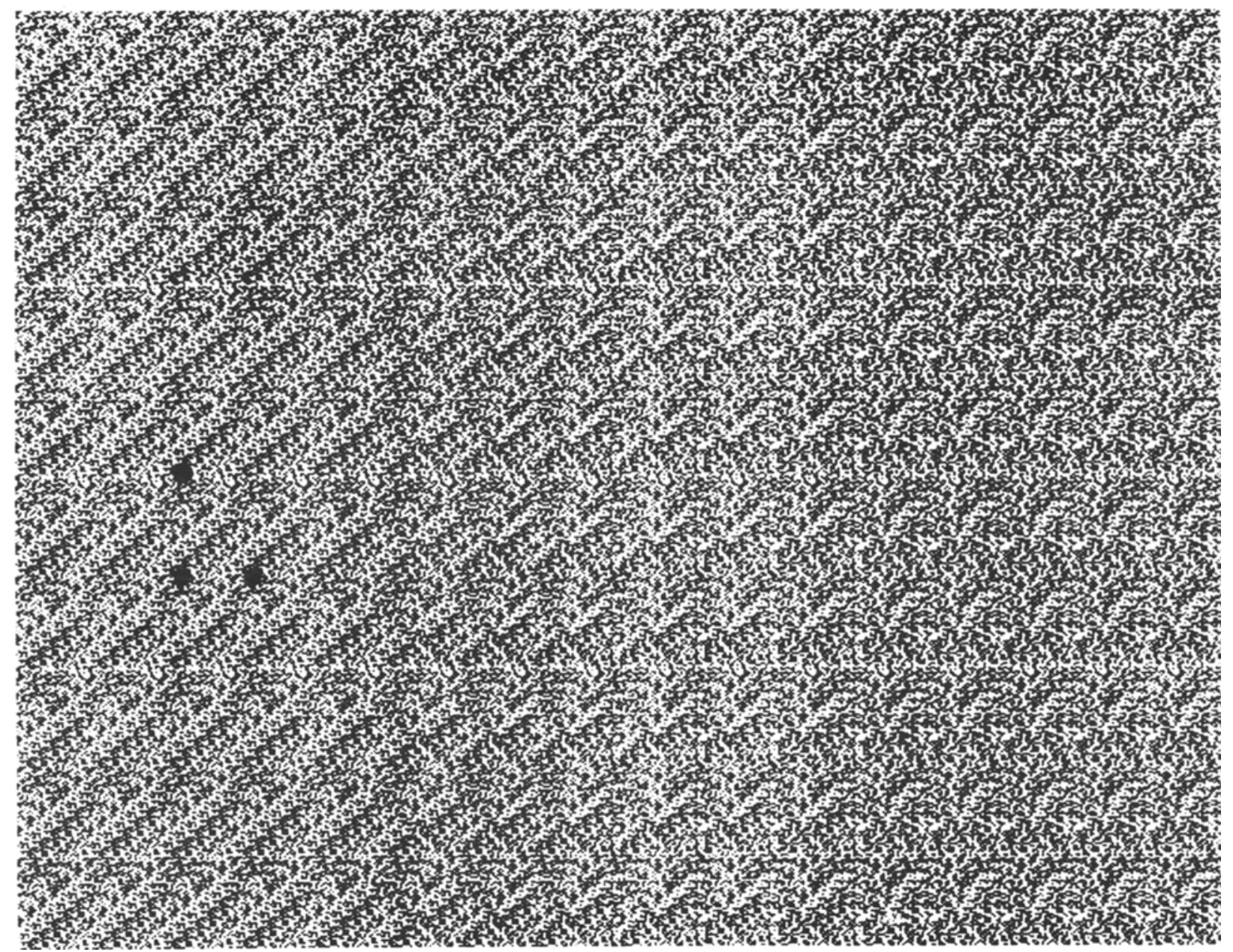

Figure 10. Ortho-ARDS. Two simple images are portrayed in orthogonal directions. Fuse the two horizontal fixation dots to see one image; then, turn the stereogram $90^{\circ}$ and re-fuse, using the heavy dots that were previously aligned vertically.

Is there an efficient means (i.e., other than explicitly calculating the cell assignments) for determining whether two arbitrary images can be portrayed in an ortho-ARDS? Likewise, given a pair of desired images, can it be shown whether or not a pair of crossing distances exists for portraying both images without ambiguities?

Unfortunately, both these questions require very subtle analysis of an extremely large number of simultaneous constraint equations, which themselves depend on the (somewhat arbitrary) choice of desired images. As such, the solution to these problems may require a novel, fresh insight into constraint satisfaction.

\section{Software}

While the algorithm outlined above for a standard ARDS is fairly straightforward, its implementation can be time-consuming and memory-intensive, since storing a depth value for each cell in even a moderate display can tax the memory allocations of a standard personal computer. Moreover, to be useful, any program should allow the user to draw the depth contours, or use images from another format in an easy and readily apparent manner.

To this end, a TrueBasic program, ARDS Plotter, has been written for Macintosh-based personal computers. Users draw the outlines of the depth planes for the desired image in MacPaint (or compatible software); then they employ the fill option to select the relative disparities of these planes. The user follows the menus in ARDS Plotter, entering the desired convergence distance, the proportion of white/black cells, whether heavy fixation dots will be presented, and so on. Figure 11 shows a simple MacPaint image and the ARDS resulting from it.

\section{Availability}

Readers wishing a copy of the program should send a blank 31/2-in. single-sided Macintosh disk and a selfaddressed return envelope to David G. Stork, Department of Psychology, Jordan Hall, Stanford University, Stanford, CA 94305. 
(a)

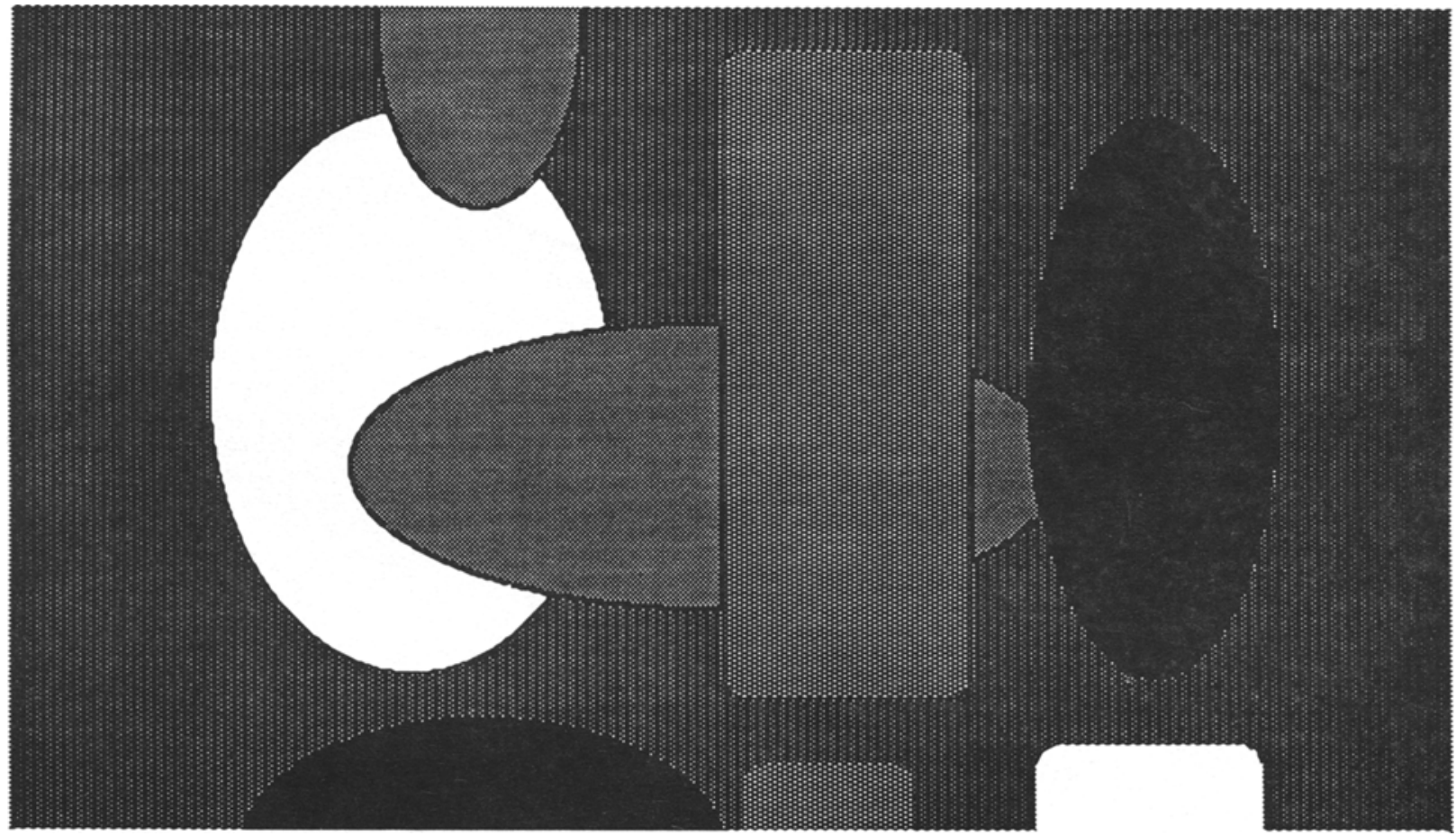

(b)

$r$ folle folit seareh format Run fonts Hindaus (2) r.

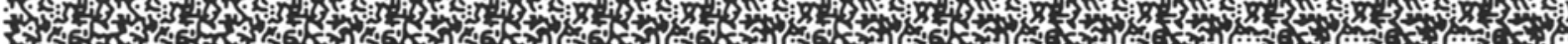
Par

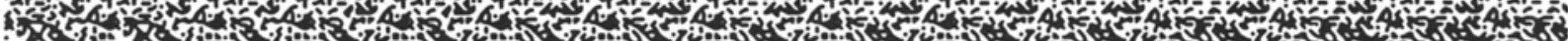
x

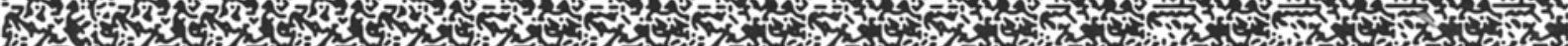

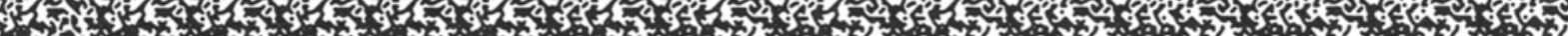
3) Z

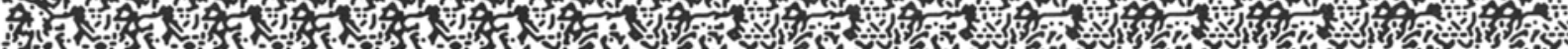
3.3. 57. H.

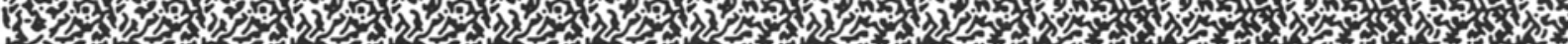
1 13.

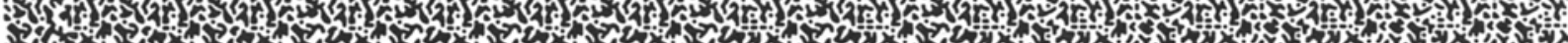
H. Ho

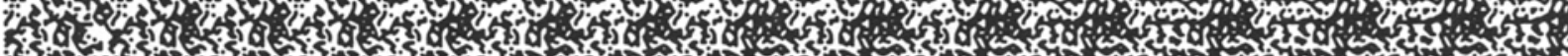
1.2. T. A 50 . F .

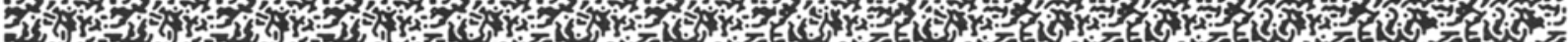
A 20 .

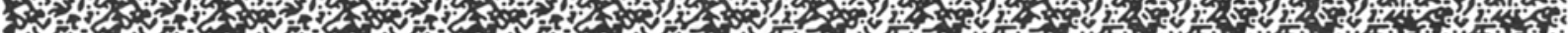
A

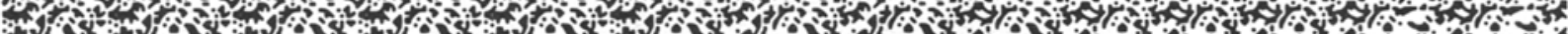
(3)

Figure 11. (a) MacPaint image sketched by the experimenter. (b) The ARDS produced by the program RDS Plotter from the image in (a). The user can choose whether or not to have the fixation dots displayed for easier viewing. 


\section{REFERENCES}

Falk, D., BrILl, D., \& STORK, D. G. (1988). Seeing the light: Optics in nature, photography, color, vision, and holography. New York: Wiley.

JULESZ, B. (1964). Binocular depth perception without familiarity cues. Science, 145, 657-658.

Julesz, B. (1971). Foundations of cyclopean perception. Chicago: University of Chicago Press.

JULESZ, B., \& CHANG, J. J. (1976). Interaction between pools of binocular disparity detectors tuned to different disparities. Biological Cybernetics, 22, 107-119.

JULESZ, B., \& SPIVACK, G. J. (1967). Stereopsis based on vernier acuity cues alone. Science, 157, 563-565.

NefF, R., SchwarTz, S., \& SToRK, D. G. (1985). Electronics for generating simultaneous random-dot cyclopean and monocular stimuli. Behavior Research Methods, Instruments, \& Computers, 17, 363-370.

Oкоsні, T. (1976). Three-dimensional imaging techniques. New York: Academic Press.

STORK, D. G., \& ABERBACH, I. (1983). Ortho-auto-random-dot stereograms. Unpublished manuscript, Swarthmore College, Department of Physics, Swarthmore, PA.

TYLER, C. W. (1974). Depth perception in disparity gratings. Nature, 251, 140-142.

TYLER, C. W. (1983). Sensory processing of binocular disparity. In C. M. Schor \& K. J. Ciuffreda (Eds.), Vergence eye movements: Basic and clinical aspects (pp. 199-295). Boston: Butterworths.
TYlER, C. W., \& RAIBERT, M. (1975). Generation of random-dot stereogratings. Behavior Research Methods \& Instrumentation, 7, $37-41$.

\section{NOTE}

1. Readers who have difficulty free-fusing should follow these directions: Place the ARDS (or a photocopy of it) flat on a table. Hold a pencil so that its tip is roughly one third of the way from the ARDS to your nose. Look at the ARDS (not the pencil)-you will see the two heavy fixation dots and two somewhat blurry pencil tips. Move the pencil (and if necessary, tip your head slightly) until both apparent tips of the pencil lie precisely at the center of each heavy fixation dot. Now hold the pencil steady, but look at the tip of the pencil. You will see a single dot at the position of the tip of the pencil, with two dots flanking on either side. Although the ARDS will be out of focus at first, relax, and in a minute or two your accommodation should naturally vary until the pattern is in crisp focus. Note that once fusion occurs, you need no longer retain the pencil or fixate the heavy dots in order to preserve proper convergence-you can inspect any point in the ARDS.

(Manuscript received January 31, 1989; revision accepted for publication August 8, 1989.) 